\title{
ARTIGO
}

DOI: https://doi.org/10.22481/praxis.v15i31.4668

\section{LO QUE SE ENSEÑANZA COMO YOGA, MINDFULNESS Y VIPASSANA EN LAS ESCUELAS POR EL MUNDO: DEBATES Y CONTROVERSIAS}

\author{
WHAT IS TAUGHT AS YOGA, MINDFULNESS AND VIPASSANA IN SCHOOLS FOR \\ THE WORLD: DEBATES AND CONTROVERSIES
}

O QUE SE ENSINA COMO YOGA, MINDFULNESS E VIPASSANA NAS ESCOLAS PELO MUNDO: DEBATES E CONTROVERSIAS

Eduardo Francisco Freyre Roach

Universidade de Havana - Cuba

Adolfo Ramos Lamar

Universidade Regional de Blumenau - Brasil

\section{Fabio Zoboli \\ Universidade Federal de Sergipe - Brasil}

\begin{abstract}
Resumen: Lo que se conoce hoy en día como yoga, mindfulness y vipassana con propósitos no religiosos, sino seculares, se ha extendido vertiginosamente por el mundo. Programas de esta índole se están introduciendo en las escuelas, prometiendo beneficios no solo para la salud, sino también espirituales: autoconocimiento, autocontrol, paz interior, conexión con el mundo, compasión, y sentido de realización plena y felicidad. Empero, cuando el programa docente se concibe en la perspectiva de la racionalidad económica neoliberal y corporativa, más bien tenderá a promover la espiritualidad del individualismo, el consumismo, la meritocracia, y la aceptación pasiva del status quo.
\end{abstract}

Palabras clave: Yoga. Mindfulness. Vipassana. Religión. Espiritualidad. Educación. Neoliberalismo.

\begin{abstract}
What is known today as yoga, mindfulness and vipassana for non-religious purposes, but secular, has spread vertiginously throughout the world. Programs of this kind are being introduced in schools, promising benefits not only for health, but also spiritual ones: self-knowledge, self-control, inner peace, connection with the world, compassion, and a sense of complete fulfillment and happiness. However, when the teaching program is conceived in the perspective of neoliberal and corporate economic rationality, it will tend to promote the spirituality of individualism, consumerism, meritocracy, and passive acceptance of the status quo.
\end{abstract}

Keywords: YOGA. Mindfulness. Vipassana. Religion. Spirituality. Education. Neoliberalism

Resumo: O que se conhece hoje em dia como yoga, mindfulness e vipassana com propósitos não religiosos, senão seculares, se extendeu vertiginosamente pelo mundo. Programas desta índole estão sendo introducidos nas escolas, prometendo benefícios não só para a saúde, senão também espirituais: 
auto-conhecimento, auto-controle, paz interior, conexão com o mundo, compaixão e sentido de realização plena e felicidade. No entanto, quando o programa docente se concebe na perspectiva da racionalidade econômica neoliberal e corporativa, em vez disso, ele tenderá a promover a espiritualidad do individualismo, o consumismo, a meritocracia e a aceitação passiva do status quo.

Palavras chave: Yoga. Mindfulness. Vipassana. Religião. Espiritualidade. Educação. Neoliberalismo.

\section{Introducción}

Lo que se conoce hoy en día como yoga, mindfulness y vipassana con propósitos no religiosos es un fenómeno de alcance global. En ciudades de las Américas, Europa y Asia, ya no es difícil encontrar gimnasios, estudios, o centros especializados en esta materia. Algunos ofrecen clases de posturas yoga (asanas), otros incluyen ejercicios con la respiración (pranayama), y técnicas de relajación. Hoy existe la tendencia de incorporar la meditación, y el yoga con otras prácticas físicas, por ejemplo, yoga pilates, yoga dance, boxing yoga etc. Más aún, existen ropas, cosméticos, suplementos nutritivos, laptop, automóviles, restaurantes, etc., con etiqueta comercial 'yoga': Lululemon's yoga pants, Lorna Jane's pants, Yoga car, Yoga lotions, Table Lenovo yoga, entre otros.

En esta perspectiva secular por mindfulness, se toma usualmente la práctica de un tipo de meditación, que estriba en concentrarse o prestar atención plena (sostenida) al flujo de sensaciones, estados de ánimos y pensamientos. Usted no interviene en ese flujo de experiencias, sino que los acepta sin juzgarlo, tal como ocurren y se manifiestan en el momento presente. Hay también productos con etiqueta: Mindful mayonesa, Mindful mint, Mindful tea.

Vipassana alude a meditación donde se practica la interiorización o introspección aguda o profunda. Mindfulness y vipassana se tratan como técnicas diferentes de meditación, pero también como sinónimos, convergentes y complementarios.

Hay lugares que ofrecen clases de asanas/pranayama yoga con mindfulness, y clases de mindfulness/pranayama que ofrecen clases de yoga asana/pranayama. Programas de este tipo se publicitan ofreciendo múltiples propósitos y beneficios, sobre todo para la salud mental y corporal, así como en el desempeño físico, deportivo, laboral y sexual. De ahí que el yoga, el mindfulness y el vipassana atraen el interés de psicólogos, neurólogos, médicos, fisioterapeutas, empresarios, periodistas etc. Los educadores físicos no son la excepción. En el contexto escolar vamos a percibir que en algunos lugares del mundo y en diferentes épocas el yoga fue y es utilizado como práctica de educación corporal. 
Frente a lo anterior, el presente ensayo pretende abordar cómo el yoga, mindfulness y vipassana se están introduciendo en las escuelas, indagando debates y controversias en lo que se refiere a las cuestiones religiosas y neoliberales que involucran esta práctica en la modernidad. Para lograr estos objetivos organizamos el texto a partir de cinco sesiones: en un primer momento presentamos el yoga, mindfulness y vipassana, en el ámbito de sus bases religiosas. En un segundo momento tratamos de la enseñanza de yoga mindfulness y vipassana sin religión en las escuelas dando foco a sus propósitos y beneficios para la salud. En la tercera parte abordamos el Yoga, mindfulness y vipassana, con propósitos y beneficios espirituales, pero sin religión. En la cuarta sesión abordamos cómo el lenguaje del self suele aparecer en la publicidad de los programas yoga en las escuelas a fin de apuntar las controversias con la ideología del yoga utilizada como producto. En la quinta y última parte colocamos las consideraciones finales del texto.

\section{Yoga, mindfulness, vipassana, y religión}

En las tradiciones religiosas de la India estos términos tienen contenido espiritual en correspondencia con la tradición religiosa que se trate: yoga en el Hinduismo, el Jainismo, y el Budismo; el mindfulness y el vipassana en el Budismo.

Los Vedas, las escrituras épicas y religiosas más antiguas que se conocen de la India, hablan de moksha, vimoksha, vimukti o mukti, es decir, de la salvación, emancipación o liberación del alma (chitta, atman,) del ciclo de reencarnaciones (samsara, karma) y su fusión o unión con el alma cósmica, suprema, universal, eterna transcendental y divina (Brahman, Purushua). Este propósito soteriológico aparece explícitamente o subyace en textos clásicos de la misma tradición como los Upanishadas, el Bhagavad Gita, los Yoga Sūtras, que se le atribuyen al sabio Patanjali, y los sutras Hatha Yoga Pradīpikā.

Entre los 196 aforismos o versos que componen los Yoga sutras, hay uno que dice que el yoga es inhibir las fluctuaciones o variaciones de la mente (Yogaś citta-vritti-nirodhah), que precisamente dificultan el alcance de moskha o, más bien quí kaivalya. Ahora bien, yoga resulta también el conjunto de Ocho Ramas, o caminos del yoga (Astangha). Dos de ellos son las asanas y pranayama. Otro es la moral, por ejemplo, abstenerse del daño y la violencia (Ahimsa). Y los demás se refieren a la absorción meditativa (samadhi, jhanas), la devoción a lo divino, actos simbólicos (mudras), y rituales (tantras). Más detalles sobre las asanas se lee en los sutras Hatha Yoga Pradīpikā. 
Entonces, lo primero que no hay que perder de vista es que en esta tradición el yoga no se reduce ni a las asanas ni pranayama ni incluso la meditación. Estos ejercicios no son fin en sí, sino que se prescriben en función de la meditación y la meta espiritual suprema, pues, desarrollan la estabilidad, el equilibrio, la salud, la templanza, energía y fuerza del cuerpo, su conexión armónica con la mente, y el sentido de relajación y realización.

Las escrituras tempranas del Budismo, el Canon pali, Tripitaka o Tipitaka, hablan de sati (termino pali) o smriti (término sanscrito), es decir, mindfulness como factor para lograr la meta religiosa suprema: Iluminación o el nibbana (nirvana), la liberación del ciclo de renacimiento (samsara, karma), de los deseos, apegos, aferramientos y sujeciones. En el Budismo no se asume que existe alma alguna (ni personal ni universal) separada y eterna (atta). Por lo tanto, de acuerdo a este postulado de la ausencia de alma (Anatta, anatma), el nibbana más bien se trata de que usted abandona esas asunciones metafísicas, y toda pretensión de auto-identificación, es decir, verlo todo en términos de esto es mío, este soy yo, este es mi verdadero ser en sí. Sati, o más exactamente samma-satti (mindfulness correcto) aparece como unos de los ocho caminos del Noble Sendero Óctuple (ariyo ațthañgiko maggo) para ese propósito espiritual supremo. En un texto budista clásico, el Satipatthana-Sutta, técnicamente hablando, mindfulness resulta la actitud o práctica de contemplar con plena atención o consciencia el cuerpo (kaya), sus partes, sus procesos, y hasta su proceso de disolución, es decir, la contemplación de un cadáver. El mindfulness se práctica en cualquier postura del cuerpo (sentado, parado, acostado, caminando...), y en cualquier actividad (comiendo, trabajando, etc.). Hay también mindfulness de la inspiración y exhalación involuntaria (anapana), de las sensaciones (vedana), de los estados mentales (citta), de la conciencia (viñana).

En el Milindapanha sutta por sati se entiende el cultivar la mente en la retención de lo atinado, lo noble, lo sano, lo correcto, y la percepción directa de lo real o verdadero en sí (HARRINGTON; DUNNE (2018), MENDIS; HORNER (1993)), es decir, el conocimiento no perturbado por la asunción de un yo en sí, alma (atta), ego o un yo, así como por los venenos (odio, codicia e ignorancia), deseos, apegos o aferramientos. Un texto budista clásico de meditación, el Arya-ratnakuta-sutra, indica que el logro meditativo solo puede alcanzarse a partir del cultivo de valores sublimes, Brahma-viharas como la compasión, el amor, la amabilidad, y la ecuanimidad. Si el yogui o la yoguini pasan por alto esto, sería como “ordenar el cuerno de una vaca". 
A ciencia cierta no se sabe si hubo face-to-face entre Buda y sus primeros discípulos, y Patanjali. Sin embargo, a pesar de las rupturas, hay elementos que hacen pensar en alguna relación de continuidad, influencia o polinización entre estas tradiciones.

Antes que todo, tómese en cuenta que el Canon pali budista es cronológicamente anterior al Gita y los Yoga sutras, y que estos textos enseñan meditación con propósitos espirituales. Es verdad que fragmentos del Canon pali se refieren implícitamente a las asanas, por ejemplo, el Satipattana sutta cuando recomienda que para practicar sati usted debe sentarse con las piernas cruzadas, buscando rectitud de la vertebra dorsal, estabilidad y equilibrio del cuerpo. Pero, allí se coloca el énfasis en que sati puede hacerse en cualquier postura del cuerpo y actividad. En la tradición védico-brahmano hinduista por asanas se incluyen posturas especiales que requieren entrenamiento. $\mathrm{Y}$ lo mismo se prescribe con respecto a la respiración cuando se habla de pranayama. El ejercicio con la respiración, anapanasati o sati de la respiración, que se recomienda en el Canon pali es más bien que usted se concentre en cómo ella ocurre espontáneamente, es decir, usted no interviene en su flujo natural.

Se conoce que Sidharta Gautama, Buda, incorporó a sus instrucciones de meditación técnicas que aprendió siendo discípulos de dos maestros yoguis samkhyas: Ramaputta y Udakka. Patanjali y Buda se inspiran en la misma tradición samkhya yoga. Probablemente, Buda y sus discípulos dominaban técnicas de asanas y pranayamas, pero con el enfoque o actitud sati. Y lo mismo vale para corrientes del Budismo en la India como los Yogacharas, así como en el Budismo del Tibet, donde se habla de yoga como meditación, pero incluyen también de asanas y pranayama, mudras, tantras, etc. No obstante, el Budismo no asume el postulado de un alma (atta) separada y eterna.

Ahora bien, en Occidente la actitud hacia el yoga y el mindfulness no es homogénea. Históricamente, y hasta nuestros días, hay quien se muestra reluctante. Por ejemplo, durante el apogeo de la colonización de la India a los yoguis se les acusaba y arrestaba por incurrir en actos blasfemos, perversos, y ofensivos (HEATER, 2014). Entre católicos que apoyan el status colonial de la India o realizan labor misionera, no era extraño el anhelo de eliminar toda índole de paganismo, heterodoxia o herejía. O que los paganos se convirtieran en cristianos.

Empero, en esa hostilidad interviene no solo este factor del diferendo religioso, sino también económico. Pues, por ejemplo, en el siglo XVIII ni a la Compañía Británica de las Indias Orientales (The East India Company) ni a las autoridades imperiales británicas les encantaba que grupos de rebeldes militantes yoguis controlaban importantes rutas comerciales 
(SINGLETONN, 2010). En estas condiciones lógicamente era impensable que se enseñara yoga en las escuelas de Occidente.

Hay cristianos reluctantes a la introducción de yoga en las escuelas, argumentando que eso no es más proselitismo religioso, lavado de cerebro o "Caballo de Troya" en suelo cristiano. Escepticismo hay entre cristianos respecto al mindfulness y al vipassana por el simple hecho de que son prácticas del Budismo, no son bíblicas, y encubren el hecho de que solo Dios y Jesús, y no el ser individual, puede darnos calma y paz mental en cualquier circunstancia de la vida (BECKER, 2015). O porque se interpreta que estas prácticas de clarificación o purificación de la mente, le abren las puertas a los pensamientos y acciones demoniacas (KOZLOWSKI, 2013).

Documentos del Vaticano acusan al movimiento de la Nueva Era (New age) de promover el yoga en la perspectiva de la sacralización del libre albedrío, el individualismo y el gnosticismo. Sin embargo, se censura la discriminación, acoso y persecución direccionada contra las religiones no cristianas ${ }^{1}$. E inclusive sugieren que la recitación cristiana puede enriquecerse con el yoga ${ }^{2}$. Otros abogan incluso por un modelo cristiano de su práctica (SYMINGTON; SYMINGTON 2012). Feligreses cristianos, e inclusive de otras religiones, no perciben que practicando yoga traicionan su religión (KIMBAR, 2016). Mientras que unos hablan de yoga cristiano, otros de alternativa cristiana al yoga, como es lo que se viene conociendo como el movimiento de Praise Moves (FLORES, 2014).

En los Estados Unidos hay quienes se quejan de que el mindfulness está ocupando el espacio escolar de la recitación antes de ser derogada en 1960 (GUNTER, 2014). A esta queja se suman tanto cristianos como quienes ven en esa enseñanza un peligro para la educación secular o laica. Existe también la sospecha de que en los programas de mindfulness en las escuelas se confundan las fronteras entre pedirles a los alumnos que sean mindful y que se sienten y se comporten.

\section{La enseñanza de yoga, mindfulness y vipassana sin religión en las escuelas: sus propósitos y beneficios para la salud}

Empero, tras la abolición oficial de colonización se intensifica el intercambio cultural entre las metrópolis europeas y la India. Aparecen quienes desean diseminar esos saberes en

\footnotetext{
${ }^{1}$ Nostra Aetate. Declaration on the relationship of the church to non-christian religions (Second Vatican Council October 28, 1965).

2 "Letter to the bishops of the catholic church on some aspects of christian meditation" del 15 de Octubre de 1989.
} 
Occidente, por considerar sus beneficios para todos independientemente de su credo religioso, o, inclusive en caso de que no profese religión alguna.

En el primer caso, y si nos ubicamos ya en la primera mitad del XX, merece que mencionemos a Tirumalai Krishnamacharya y Paramahansa Yogananda. Después, Swami Vivekawanda que en los 50, presenta en Inglaterra y en los Estados Unidos el yoga (el RajaYoga) como meditación espiritual, que nos invita a observar, controlar, e iluminar nuestros estados internos, sensaciones o pensamientos. Su proyecto de compartir el conocimiento del yoga en Occidente arranca de la siguiente premisa que el expresara en el famoso discurso en el Parlamento de las Religiones celebrado en Chicago en 1893:

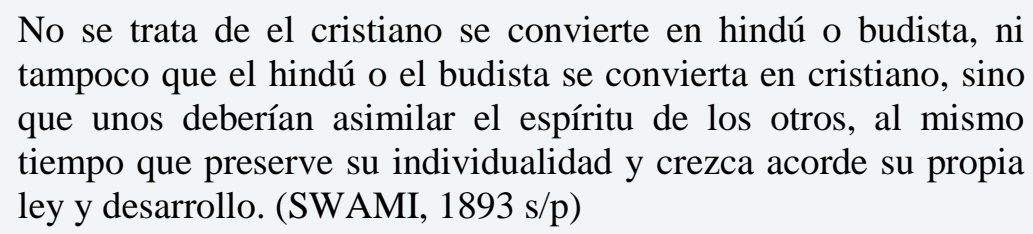

Estudiosos del papel de Vivekananda en la diseminación del yoga en Occidente, reclaman considerar que el yoga no estaba en su plan, sino que fue entrando en el mismo en respuesta de demandas de esa enseñanza en los Estados Unidos.

Uno de los discípulos de Krishnamacharya, B.K.S Iyengar, entre 1954 y 1970 populariza un yoga centrado en las asanas y pranayama y sus beneficios terapéuticos, pero sin contenido espiritual religioso alguno. Gracias a su carisma y el apoyo del Ministro de Educación Física y Deportes Peter McIntosh, el yoga de Iyengar ganó institucionalización a partir de 1979 (HUGGINS, 2001) y hoy en día tiene gran audiencia en todo el mundo. El efecto de lo que autores, siguiendo a Max Weber, llama la rutinización del carisma ('the routinization of carisma), favoreció más a B.K.S Iyengar que a Yogini Sunita, la cual veía el yoga como un camino de vida, como actividad cotidiana, y no le interesaba integrar la práctica del yoga en el sistema de cálculo institucional y burocrático (NEWCOMBE, 2014).

La propuesta de un mindfulness secular más ambiciosa, sistemática, y de gran apoyo institucional y corporativo son los programas basados de lo que desde la década del 70 se conoce como Mindfulness-Based Stress Reduction (MBSR). Kabat-Zinn, el pionero de este campo, suele definir el mindfulness en términos de prestar atención consciente a las experiencias que pasan por nuestra mente en el momento presente, aceptándolas cómo son, sin juzgarlas, sin aferramiento, y permitiendo que se disipen. Autores se refieren a que Kabat Zinn adoptó parte de la definición de 'sati' que Rhys David propuso cuando en 1890 tradujo el Milindapanna sutta (HAFFERMAN, 2015) que Rhys Davids propuso definir sati como 
'atention', 'mindfulness'. Otros autores explican que en ese sutta, el mindfulness se define como una de las facultades espirituales, y llaman la atención que allí mindfulness significa más bien una especie de llamar a la mente los dharmas que participan en lo que es sano o malsano, deseable o repudiable, sublime o inferior (RHYS-DAVIDS, 1890; HARRINGTON; DUNE, 2018, MENDIS; HORNER, 1993). No hay puesto para este aspecto espiritual en la definición de mindfulness que propone el fundador del campo de MBSR, Kabat Zinn.

Actualmente en Estados Unidos, el Reino Unido, Canadá entre otros países se enseña en las escuelas yoga, mindfulness y vipassana a lo secular, auguran múltiples beneficios terapéuticos, neuronales, cognitivos, conductuales y académicos, tanto para los estudiantes como sus maestros. Se suele argumentar la eficacia de esos programas, apelando a resultados de investigación en Psicología y Neurología, así como testimonios, y experiencias docentes (BUTZAR; EBERT; TELLES; KAHALSA, 2015; DAVIDSON; DUNNE; ECCLES, 2012; SEMPLE; VITA; BRITTANY, 2017).

\section{Yoga, mindfulness y vipassana con propósitos y beneficios espirituales, pero sin religión}

Puede observarse fácilmente que abunda los programas de reapropiación secular del yoga, el mindfulness y el vipassana, que coloca el énfasis en los propósitos y beneficios exclusivamente para la salud corporal y mental. Sin embargo, si antes se excluía todo contenido espiritual, hoy en día, es muy natural que los programas de yoga, mindfulness y vipassana ofrezcan propósitos y beneficios espirituales, aunque no de carácter religioso.

Por ejemplo, Iyengar (2016), cuyo yoga sin espiritualidad y sin religión alcanzó gran popularidad, ahora ha declarado que su motivación en diseminar la práctica del yoga, siempre ha sido espiritual. Inclusive ha sentenciado recientemente que hacer asanas y pranayama, volteando la mirada hacia las normas de conducta y la moralidad, es 'mera acrobacia'.

The Seven Spiritual Laws of Yoga de Deepak Chopra y David Simon es la plataforma de instrucción en yoga que ofrece el Chopra Centre y sus filiares diseminadas por todo el mundo. El postular espiritual básico estriba en que a través de la realización de las asanas, usted logre la unidad armónica cuerpo, mente, y espíritu. Y este beneficio espiritual comienza con que el practicante asuma que su ser o yo esencial es pura conciencia, fuente infinita de toda existencia en el mundo físico, y por lo tanto, usted es creatividad infinita, sin ataduras, y eterno. Le sigue la proyección del amor, la felicidad, el éxito, la realización de sus deseos, y magnificas relaciones con los demás. 
Kabat-Zinn, el principal promotor de este campo de MBSR, hoy está diciendo que está evitando hablar de mindfulness secular, abstraído de lo sacro o sagrado. Pero insiste en que Buda no tenía la intención de fundar una religión, sino erradicar el sufrimiento mental (BAER, 2017).

La organización Mindful Schools en Oakland muestra en su Website dice que, mindfulness mejora también el sentido de compasión y compromiso social ${ }^{3}$. Se apuesta incluso a que la enseñanza del yoga y el mindfulness contribuya a la educación holística de los niños.

El Vipassana Meditation Center in Hong Kong Sheung Shui promete que la técnica Anapata desarrolla en los niños y adolecentes concentración, paz, calma, perseverancia, amabilidad y amor, y, en fin, 'to be better persons'. El Código de Conducta exige durante el curso hacer voto, repetir y cumplir preceptos del Noble Camino Óctuple tales como la abstención de hacer daño, quitar la vida, robar, conducta sexual inapropiada, mentir, ofender, e ingerir bebidas alcohólicas.

Ahora bien, es importante no perder de vista que abundan programas de enseñanza yoga, el mindfulness y vipassana con propósito de lucro corporativo. Billonario es el negocio del yoga y el mindfulness en los Estados (HANNAH, 2018). Se incluye no sólo la venta de clases presenciales (face-face y online) y artículos (ropa, equipos, accesorios) de uso en la práctica de yoga. Se venden artículos (ropa, cosmético, suplementos alimenticios, etc., con marca o étiqueta yoga o mindfulnes, pero con otros propósitos. Lululemon's yoga pants, Lorna Jane's pants, Yoga car, Yoga lotions, Table Lenovo yoga, Mindful mayonesa, Mindful mint, Mindful tea etc.

Dado a esta orientación al lucro no es sorpresa que corporaciones del yoga reclamen copyright sobre la secuencia de asanas o ejercicios de pranayama. Es el caso de corporaciones como Bikram yoga y Phillipines-based Institute for Inner Studies, y otras, que han reclamado copyright sobre sus técnicas. Kabat-Zinn ostenta marca comercial sobre el tratamiento de $\mathrm{MBRS}^{4}$. Claro, la protección de derecho de autor y la protección de las marcas trae beneficios comerciales. Sin embargo, aunque el gobierno de la India, estipulan medidas que desafían la actitud de privatización del yoga5, hoy en día hay legislaciones que contemplan cierto

\footnotetext{
${ }^{3}$ Research on Mindfulness.Mindful shools. https://www.mindfulschools.org/about-mindfulness/research/

4 Coyne James. What Jon Kabat-Zinn's trademarking of MBSR means for mindfulness training. https://jcoynester.wordpress.com/2017/05/19/what-jon-kabat-zinns-trademarking-of-mbsr-means-formindfulness-training/

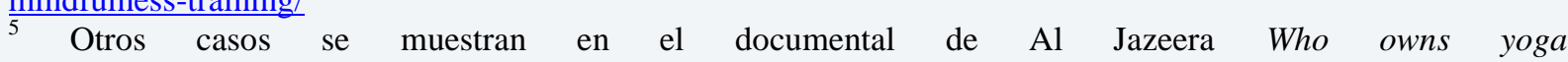
(https://www.youtube.com/watch?v=ZmJNsvc63pM ). También puso en marcha un proyecto bibliotecario (La
} 
derecho intelectual sobre actividades de yoga que cabrían dentro del concepto de work of dance art, es decir, arte de la dance (German Copyright Act) o corografía, choreographic works o choreographic authorship (BENJAMIN; KONSTATIN, 2017).

Este negocio corporativo del yoga, el mindfulness y vipassana, y su replicación en escuelas forma, parte del status quo neoliberal hegemónico, donde se promueven la liberalización, privatización, mercantilización, y, por lo tanto, la racionalización económica de servicios públicos como la salud y la educación. Los trabajos de filósofos como Heidegger, Foucault, Habermas y Max Weber, aportan elementos que caracterizan esa racionalidad.

Por ejemplo, un curso de mindfulness se publicita resaltando aspectos como: Eficacia (autoayuda, reducción de la depresión, la adicción, y el dolor tanto físico como mental, y el bienestar), sin importar las diferencias personales; predicción y calculación (resultados del programa entre 5 y 12 horas); y Control técnico no humano de las personas (para hacerlas mejores negociantes, empleados, consumidores, educandos, soldados) (TERRY, 2017). Cursos de yoga y vipassana son concebidos y evaluados con estos criterios de racionalidad económica instrumental. Un programa escolar de yoga, mindfulness y vipassana montado en esta lógica, tenderá la instrumentación y estandarización del currículo, los maestros, y los alumnos. Esta simplificación se manifiesta de forma más acentuada cuando se coloca el énfasis en que con un curso de yoga, mindfulness o vipassana usted aprenderá a conocerse a sí mismo, y logrará la capacidad de ayudarse, y desarrollar paz mental, realización personal, y felicidad verdadera.

Por ejemplo, estudios Yoga Basics, dirigidos a principiantes o novatos, se presentaban con el slogan aeróbico de los 70 y los 80: ¡No pain, no gain!, ¡Sin dolor, no se gana!; igo for the burn!, ¡quema calorías! Pero ya desde los 90 pasa a primer plano el slogan ¡Listen to your body, go at your own pace!, es decir, presta oídos a tu cuerpo; ¡to move at your own pace!, ¡refúgiate en tu paz interior!; ¡do not push yourself!, ¡no se maltrate!; ¡exploring selfcompassion!, ¡explora la compasión en ti mismo! (BRYANT, 2014). El lenguaje del 'self', es decir, del yo, el en sí mismo, tiene gran presencia en la publicidad de las ofertas de clases de yoga que ofrece la corporación Yoga Basics, y otras corporaciones. En su informe de 2016 de Yogipreneur y Yoga Journal ${ }^{6}$ se dice que el goce que reporta, su impacto en la salud, y la disminución del stress, son las tres razones fundamentales para practicar yoga. Se reporta que

Traditional Knowledge Digital Library) que libera el acceso universal a ese saber, y hasta ha creado un Ministerio del Yoga que atienda todo lo relacionado.

${ }^{6}$ Vea: http://www.yogajournal.com/category/teach/business-of-yoga/ 
comparado con la población en general, $20 \%$ de quienes hacen yoga dicen tener imagen positiva de sí mismo (a positive self-image).

Puede seguirse los rastros de esta especie de en la publicidad del yoga en los centros B.K.S Iyengar; Yoga Basics; Bikram yoga; Pure yoga; Yoga Alliance; Yoga Journal; Chopra centers, Yogapreneur, y en los escritores New Age (Georgeanne Lamont, Tom Beaudoin, Sharon Janis, Osho Rajneesh, Jesper Kunde, John Grant, Morgan Scott Peck, Deepa Chopra, y otros). Autores alertan que este tipo de Spirituality of the Self, de espiritualidad del sí mismo, expresa el reacomodo de los saberes orientales a los intereses del neoliberalismo corporativo. Sus componentes son:

Atomización: la individualización de la responsabilidad sin consideración de la sociedad.

Interés propio: una ética de interés propio que considera el beneficio como la motivación principal para la acción humana. Corporativismo: ubicar el éxito corporativo (no comunitario) por encima del bienestar y la seguridad laboral de los empleados. Utilitarismo: tratar a los demás como un medio en lugar de como un fin (por ejemplo, ver a los humanos como consumidores para persuadirse, a otros negocios como competidores a superar, o a los empleados como recursos para ser utilizados). Consumismo: la promoción de la satisfacción desenfrenada del deseo como la clave de la felicidad. Quietismo: aceptación tácita o abierta de la inevitabilidad de la injusticia social en lugar de un deseo de superarla. Miopía política: un reclamo de neutralidad política: la negativa a ver las dimensiones políticas de la "espiritualidad". Control del pensamiento / acomodamiento: uso de técnicas psicofísicas, descritas en términos de "desarrollo personal", que buscan pacificar los sentimientos de ansiedad e inquietud a nivel individual en lugar de tratar de desafiar las desigualdades sociales, políticas y económicas que causan tales angustias. (CARRETTE; KING, 2005, p.21-22).

\section{El lenguaje del self suele aparecer en la publicidad de los programas yoga en las escuelas $^{7}$}

Hay alertas sobre el carácter racial, sexista, elitista, meritocratico, y colonial del yoga y el mindfulness a lo comercial y corporativo. Por ejemplo, se ve preocupación que la enseñanza del yoga y el mindfulness sutilmente desliza la imagen de que el yoga es para personas blancas, delgadas, y de mucho dinero (MULPHY, 2014). Denuncias hay sobre los programas de mindfulness están dirigidos principalmente a las escuelas donde predominan

\footnotetext{
${ }^{7}$ Para conocer más sobre programas de yoga para en las escolas consultar: "BUTZAR, B.; EBERT, M.; TELlES, S.; KHALSA, S. B. S. School-based Yoga Programs in the United States: A Survey Adv Mind Body Med. 2015." y "HYDE, A. The yoga in schools movement: using standards for educating the whole child and making space for teacher self-care. In: GORLEWSKI, J.A.; PORFILIO, B. J.; GORLEWSKI, D. A, editors. Using Standards and High-Stakes Testing for Students: Exploiting Power with Critical Pedagogy. Peter Lang Publishing; New York, NY: 2012. pp. 109-126”.
} 
alumnos 'de color', y con el propósito de predisponerlos a la pasividad y la aceptación de la situación.

Se habla mucho que el yoga y el mindfulness mejorar la capacidad de liderazgo y prosperidad en los negocios. Y se citan corporaciones que están introduciendo yoga y mindfulness para directivos y empleados (bancos, empresas etc.).

Otra estrategia meritocrática es las citaciones de declaraciones de celebridades que asocian su éxito con la práctica del yoga y el mindfulness, por ejemplo: Jane Fonda, Jennifer Aniston, Julia Roberts, Lady Gaga, David Beckham, Obama y su esposa, Katy Perry, Paul McCartney, Madonna, Hugh Jackman, and Clint Eastwood, Tina Turner, Steve Jobs, Emma Watson, Davina McCall, Angelina Jolie y Oprah Winfrey, entre otros.

Por el precio de las clases de yoga las cadenas transnacionales de gimnasios y estudios son accesibles para personas de clase media y rica. Para que se tenga una idea, una hora de clase suele valer 10 euros, y 40 euros si es clase privada. Estudios sugieren que entre el 2013 y el 2018 el precio de las clases de yoga de una hora, oscilan entre 10 y 30 euros o dólares ${ }^{8}$. Las clases de mindfulness suelen costar alrededor de 20 US por 30 minutes. Un curso de 30 días de MBRS vale de 420 a 570 US.

Los “yoga pants" para mujeres están hoy de moda. Se apela a un lenguaje que llama la atención hacia la reafirmación de la individualidad, la exclusividad, la belleza corporal, la imagen sexual, y status social. Empresas de ropa como Lululemon publicita yoga pants para muchachas, asegurando que esos yoga pants las hará sentir de confiada y sexy. Sloganes como "yoga pants for women hot" es un ejemplo de esa estrategia.

Unos denuncian que tal reapropiación secular desnaturaliza y distorsionan esos saberes, con vistas a la acumulación incremental de ganancias. De ahí que faltan quienes reclaman el cese de la enseñanza de yoga secular en las escuelas y universidades ${ }^{9}$, pues ven en ello un gesto de opresión y genocidio cultural.

\section{Consideraciones finales}

El state of the art en materia del debate y las controversias sobre la introducción de clases de yoga y de mindfulness en las escuelas permite decir que ciertamente, en el contexto de los sistemas de educación secular, no sorprende que tenga receptividad la enseñanza

\footnotetext{
${ }^{8}$ Yoga Prices: How Much Should You Charge for Classes? https://www.yogabaron.com/yoga-prices

9 https://www.washingtonpost.com/news/morning-mix/wp/2015/11/23/university-yoga-class-canceled-becauseof-oppression-cultural-genocide
} 
secular del yoga y el mindfulness, es decir, que se excluye todo propósito y contenido docente de carácter espiritual y religioso. Empero, hoy en día, se está promocionando la enseñanza del yoga, el mindfulness y el vipassana con contenido espiritual, aunque no religioso.

Se observa que abundan los programas de esa enseñanza montada en los imaginarios de la racionalidad instrumental, gobernabilidad, subjetividad, educación, cultural, y espiritualidad, que promueve el status quo neoliberal y corporativo hegemónico y global. Pueden sucumbir a esta tendencia las clases de yoga y mindfulness, aunque no estén orientadas al lucro, dependan de donaciones, y ofrecen acceso masivo.

La introducción de la espiritualidad en los programas de yoga y mindfulness en los negocios y en educación plantean el problema de su medición, es decir, de si la espiritualidad y su impacto en la salud, en la educación, etc., es medible, instrumentalizable, operacionalizable, estandarizable, y mediante qué variables. El debate que hoy existe al respecto gira en torno a la conceptualización de la espiritualidad en términos de asunciones religiosas y metafísicas, o relativas al bienestar, y su universalidad.

Pero salta a la vista que las supuestas variables que conforman la espiritualidad no son susceptibles de medición, instrumentación, y estandarización, como en el caso de ciertos indicadores físicos, mentales, o de desempeño físico. Por otro lado, si bien un curso puede incluir fácilmente perspectivas espirituales, por ejemplo, lo relativo a los valores, resulta poco probable que el empaquetamiento hiper-individualista del curso lo permita (KRZNARIC, 2017).

Se sabe muy bien que la transformación espiritual- en lo que respecto, por ejemplo, a como uno se percibe en sí mismo y percibe al mundo- es largo plazo, y en dependencia del temperamento, y las características personales.

La espiritualidad del yoga, el mindfulness y la vipassana en las tradiciones religiosas de la India no propone el énfasis en la individualidad o self como fin en sí, sino todo lo contrario, desafían a los programas que incentivan el egocentrismo, el consumismo, y el elitismo.

\section{REFERÊNCIAS}

BAER, D. The Father Of Mindfulness on What Mindfulness Has Become An interview with Jon Kabat-Zinn, creator of Mindfulness-Based Stress Reduction. Trivel Global, April 12, 2017. Disponible en: https://medium.com/thrive-global/the-father-of-mindfulness-on-whatmindfulness-has-become-ad649c8340cf 
BECKER, A. J. Has Mindfulness Supplanted Thoughtfulness? CT Christianity Today. April 20, 2015. Disponible en: https://www.christianitytoday.com/amyjuliabecker/2015/april/hasmindfulness-supplanted-thoughtfulness.html

BENJAMIN, B.; KONSTATIN, V. W. Yoga and copyright. Wipo Magazine. June 2017. Disponible en: http://www.wipo.int/wipo_magazine/en/2017/03/article_0008.html

BRYANT, K. The art of practicing self compassion. Yoga basics, March 25th, 2014. Disponible en: http://www.yogabasics.com/connect/yoga-blog/the-art-of-practicing-selfcompassion/

BUTZAR, B.; EBERT, M.; TELLES, S.; KHALSA, S. B. S. School-based Yoga Programs in the United States: A Survey. Adv Mind Body Med. Fall; 29(4): 18-26, 2015. Disponible en: https://www.ncbi.nlm.nih.gov/pmc/articles/PMC4831047

CARRETTE, J.; KING, R. Selling Spirituality: The Silent Takeover of Religion. Routledge, New York, 2005.

DAVIDSON, R. J.; DUNNE, J.; ECCLES, J. S. Contemplative practices and mental training: prospects for American education. Child D ev Perspect. V. 6(2):146-153, 2012.

FLORES, T. PraiseMoves reignites debate over yoga's nature. Washington Times, August 2, 2014. Disponible en: https://www.washingtontimes.com/news/2014/aug/2/praisemovesreignites-debate-over-yogas-nature/

GUNTER, B. C. Mindfulness Meditation in Public Schools: Side-Stepping Supreme Court Religion Rulings. The Blog 12/05/2014 04:18 pm ET Updated Dec 06, 2017. https://www.huffingtonpost.com/candy-gunther-brown-phd/mindfulness-meditationin_b_6276968.html

HAFFERMAN, V. The Muddied Meaning of 'Mindfulness'. The New York Times Magazine. 14 Abril 2015. Disponible en:

https://www.nytimes.com/2015/04/19/magazine/the-muddied-meaning-of-mindfulness.html

HANNAH, H. K. The Meditation Industry. SAGE: business researcher January 29, 2018.

Disponible en: http://businessresearcher.sagepub.com/sbr-1946-105603-

2878495/20180129/the-meditation-industry

HARRINGTON, A.; DUNNE, J. Forthcoming. Mindfulness meditation: Frames and choices. American Psychologist. August 13, 2018 10:27:02 AM EDT. Disponible en: http://nrs.harvard.edu/urn-3:HUL.InstRepos:10718406

HUGGINS, M. Walking in the Footsteps of a Pioneer: Peter McIntosh - TrailBlazer in the History of Sport. International Journal of the History of Sport, 18 (2), pp. 1 36- 1 47, 2001.

IYENGAR, B. K. S. The Yoga Master at Ninety, an Interview with B.K.S. Iyengar June 21, 2016. 
KIMBAR, K. Can Catholics do Yoga? Post.Kitchen Stewardship. 2016. Disponible en: https://www.kitchenstewardship.com/can-catholics-do-yoga/

KOZLOWSKI, E. “Can Christians Practice Mindfulness?” Huffpost. The blog. 05/07/2013 04:31 pm ET Updated Jul 07, 2013. Disponible en: https://www.huffingtonpost.com/edenkozlowski/mindfulness-and-religion_b_3224505.html

KRZNARIC, R. How We Ruined Mindfulness. Time, May 26, 2017. Disponible en: http://time.com/4792596/mindfulness-exercises-morality-carpe-diem/

MENDIS, N. K. G.; HORNER, I. B. The questions of King Milinda: an abridgement of the Milindapañha. Buddhist Publication Society, 1993.

MULPHY, R. Why Your Yoga Class Is So White. The Atlantic, July 8, 2014. Disponible en: https://www.theatlantic.com/national/archive/2014/07/why-your-yoga-class-is-sowhite/374002/

NEWCOMBE, S. The institutionalization of the yoga tradition: "gurus" B. K. S. Iyengar and Yogini Sunita in Britain. In: SINGLETON, M.; GOLDBERG, E. (eds.) Gurus of Modern Yoga. Oxford University Press, New York, USA, pp. 147-167, 2014.

RHYS-DAVIDS, T. W. The questions of King Milinda. Oxford: The Clarendon press, 1890.

SEMPLE, R. J.; VITA, D.; BRITTANY, A. R. Mindfulness goes to school: things learned (so far) from research and real-world experiences. Psychology in the schools 54.1 (2017): 29-52. PMC. Web. 13 Aug. 2018.

SINGLETON, M. Yoga Body. The Origins of Modern Posture. Oxford University Press, 2010.

SYMINGTON, S. H.; SUMINGTON, M. F. A Christian Model of Mindfulness: Using Mindfulness Principles to Support Psychological Well-Being, Value-Based Behavior, and the Christian Spiritual” Journey. Journal of Psychology and Christianity;

Atavia Vol. 31, Iss. 1, 2012. Disponible en:

https://search.proquest.com/openview/75721d4697652039de0ddcae1a124764/1?pqorigsite $=$ gscholar $\& \mathrm{cbl}=38088$

SWAMI. Vivekananda's speech at the Parliament of Religion, Chicago in 1893: Full text". The Indian Express, Sunday, Septembre02, 2018. Disponible en:

https://indianexpress.com/article/india/pm-narendra-modi-swami-vivekanandas-speech-at-theparliament-of-religion-chicago-in-1893-full-text-4838148/

TERRY, H. McDonaldizing Spirituality: Mindfulness, Education and Consumerism. Journal of Transformative Education, March 2017. Diponible en:

https://www.researchgate.net/publication/315109433_McDonaldizing_Spirituality_Mindfulne ss_Education_and_Consumerism

TLALKA, S. Meditation in Public Schools: Pro or Con? Mindful. Healthy mind, healthy life. May 12, 2017. Disponible en: https://www.mindful.org/meditation-public-schools-pro$\underline{\mathrm{con} /}$ 


\section{$\underline{\text { SOBRE OS AUTORES }}$}

\section{Eduardo Francisco Freyre Roach}

Doutorado em Ciências Filosóficas, pela Universidad Estatal Lomonosov de Moscu (UELM), na Rússia. Pós doutor em Educação pela Universidade de Campinas (UNICAMP). Professor da Universidade de Havana - Cuba. E-mail: freire.roach2016@gmail.com

\section{Adolfo Ramos Lamar}

Doutora em Educação pela Universidade de Campinas (UNICAMP). Pós-Doutor em Educação pela Universidade de São Paulo (USP). Professor do Programa de Pós-graduação em Educação pela Fundação Universidade Regional de Blumenau (FURB). E-mail: ajemabra@yahoo.com.br

\section{Fabio Zoboli}

Doutora em Educação pela Universidade Federal da Bahia (UFBA). Pós-doutor em Educação do Corpo pela Universidad Nacional de La Plata - UNLP/Argentina. Professor do Programa de Pós-graduação em Educação da Universidade Federal de Sergipe (UFS). E-mail: zobolito@gmail.com 